\title{
Efficacy of surgeon-directed postoperative local injection with an analgesic mixture in posterior fusion surgery for adolescent idiopathic scoliosis
}

Hiroto Makino*, Shoji Seki, Katsuhiko Kamei, Yasuhito Yahara and Yoshiharu Kawaguchi

\begin{abstract}
Background: Severe postsurgical pain in posterior spinal fusion is common. Multimodality analgesia, including opioid-based patient-controlled analgesia (PCA), is commonly used, but opioid-related adverse events such as nausea and vomiting are sometimes a problem. We used a ropivacaine-epinephrine-dexamethasone mixture given as onetime local bilateral submyofascial injections at the operated levels added to conventional multimodality analgesia including PCA for postoperative pain control in one group of patients to confirm whether administration of this mixture reduced postoperative pain and opioid use status post posterior spinal fusion.
\end{abstract}

Methods: We retrospectively reviewed 67 consecutive patients who had undergone posterior fusion surgery for adolescent idiopathic scoliosis (AIS), 35 of whom were treated with conventional analgesia that consisted mainly of PCA (control group) and 32 of whom were treated with one-time submyofascial injections of a ropivacaine-epinephrinedexamethasone mixture (submyofascial injection group) added to conventional multimodality analgesia. We compared postsurgical pain levels and the amount of opioid use over the first $48 \mathrm{~h}$ after surgery, as well as physical activity levels and adverse events 2 weeks after surgery.

Results: Postsurgical pain quantified by a numeric rating scale (1-10) in the submyofascial injection group was significantly lower than that in the control group. The amount of fentanyl use was significantly less in the submyofascial injection group at $24 \mathrm{~h}, 48 \mathrm{~h}$, and all subsequent periods after surgery. In addition, Walking Recovery Time (WRT) defined as the number of days until the first event of ambulation was significantly less in the submyofascial injection group ( $3.3 \mathrm{~d}$ vs $4.1 \mathrm{~d}, P=0.0007)$ ). Laxative use was significantly less in the submyofascial injection group ( 0.3 times vs 1.3 times, $P=0.02$ ).

Conclusions: One-time submyofascial injections at the operated levels with a ropivacaine-epinephrine-dexamethasone mixture after spinal fusion surgery reduced pain, opioid consumption, and opioid-related adverse events. This technique can contribute significantly to postoperative analgesia.

Keywords: Adolescent idiopathic scoliosis, Spinal fusion, Postoperative pain, Opioids, Nonopioid analgesia, Ropivacaine, Epinephrine, Dexamethasone, Nausea and vomiting, Constipation, Patient-controlled analgesia

*Correspondence: hiroto@med.u-toyama.ac.jp

Department of Orthopaedic Surgery, University of Toyama, Faculty

of Medicine, 2630 Sugitani, Toyama, Toyama 930-0194, Japan

\section{Background}

Adolescent idiopathic scoliosis (AIS) is a relatively common disease. Depending on race, it is found in approximately $0.5-4 \%$ of the population [1]. Since the curvature original author(s) and the source, provide a link to the Creative Commons licence, and indicate if changes were made. The images or other third party material in this article are included in the article's Creative Commons licence, unless indicated otherwise in a credit line to the material. If material is not included in the article's Creative Commons licence and your intended use is not permitted by statutory regulation or exceeds the permitted use, you will need to obtain permission directly from the copyright holder. To view a copy of this licence, visit http://creativecommons.org/licenses/by/4.0/. The Creative Commons Public Domain Dedication waiver (http://creativecommons.org/publicdomain/zero/1.0/) applies to the data made available in this article, unless otherwise stated in a credit line to the data. 
in AIS sometimes progresses, and may cause dysfunction and back pain, bracing of curvatures $>25^{\circ}$ is usually undertaken. Surgery is always indicated for curvatures $\geq 40^{\circ}$ [2]. Posterior fusion surgery is the most common procedure for AIS. It is associated with severe postoperative pain $[3,4]$. Treatments for this pain include intravenous patient-controlled analgesia (IV-PCA), epidural anesthesia, and acetaminophen and non-steroidal antiinflammatory drugs (NSAIDs). However, many postoperative patients still experience moderate pain, and some adverse events from the analgesics may occur, especially in the case of opioids, which are associated with postoperative nausea and vomiting (PONV) and constipation. These are associated with longer length of hospital stay and higher cost of care [5]. Some recent studies have shown positive effects of local infiltration of analgesics for postsurgical pain in posterior spinal fusion [6-8].

We developed a one-time local submyofascial injection procedure with a ropivacaine-epinephrine-dexamethasone mixture for postoperative analgesia. This method is easy to apply and combine with other analgesic methods such as IV-PCA. The purpose of the present study was to confirm whether this method reduced postsurgical pain and opioid use.

\section{Materials and methods Data collection}

A total of 67 consecutive patients underwent posterior fusion surgery for AIS from January 2016 to March 2019. The electronic health record was used for data collection. From this data source, we extracted information on age, gender, height, weight, range of fusion, Numeric Rating Scale (NRS) of pain after surgery (range; 0-10), the amount of fentanyl used by PCA, walking recovery time (WRT) defined as the number of days until the first event of ambulation, and adverse events that may have been associated with postoperative analgesics including constipation, nausea, surgical site infection, and pseudarthrosis.

\section{Surgical methods}

All patients underwent surgery by a single surgeon (S.S.) at a single hospital. To briefly describe the previously reported surgical procedure [9], surgery was performed under general anaesthesia in the prone position. After pedicle screw insertion, the articular cartilage of the facet joints was removed. A Ponte procedure [10] was performed at three levels near the apex of the curvature. A concave rod $(5.5 \mathrm{~mm} \mathrm{CoCr})$ was rotated $90^{\circ}$ to transform the scoliosis into a thoracic kyphosis or a lumbar lordosis depending on the type of the scoliosis [9]. Then a convex rod was inserted, and the rib hump was reduced using a differential rod-contouring technique followed by segmental direct vertebral rotation of each vertebra [11]. Spinal cord monitoring of motor and somatosensory evoked potentials was performed during surgery.

\section{Postoperative management}

After surgery, intravenous patient-controlled anesthesia (IV-PCA) with fentanyl diluted with saline was used in all patients. The lockout time of the PCA pump was $15 \mathrm{~min}$. All patients were allowed to take oral analgesics such as NSAIDs (patients $>15$ y) or acetaminophen (patients $<15$ y) from next day after surgery. In addition, they were also allowed to use rectal analgesics or intravenous acetaminophen as needed. The submyofascial drain was removed $3 \mathrm{~d}$ after surgery and patients were allowed to stand and walk as their pain allowed. Metoclopramide for nausea and laxatives for constipation were used as needed. The patients undergoing surgery between January 2016 and December 2017 were treated with this analgesic protocol (control group), while the patients after January 2018 were additionally treated with a one-time postoperative ropivacaine-epinephrine-dexamethasone mixture injection (submyofascial injection group, Fig. 1).

\section{Local injection}

with a ropivacaine-epinephrine-dexamethasone mixture We used a mixture of ropivacaine $(3 \mathrm{mg} / \mathrm{kg})$, epinephrine $(0.003 \mathrm{mg} / \mathrm{kg})$, and dexamethasone $(0.66 \mathrm{mg} / \mathrm{kg})$, diluted up to $50 \mathrm{~mL}$ with saline. Before myofascial closure, bilateral $1-2 \mathrm{~mL}$ submyofascial injections of the mixture were administered at the operated levels (Fig. 2).

\section{Statistical analysis}

Data are shown as the mean and standard deviation. We used a two-way repeated measures ANOVA followed by the Tukey-Kramer post-hoc test for comparative analyses of NRS. Student's $t$-test was used to compare the demographics and the results of the amounts of opioids needed, the WRT, the number of times an antiemetic was needed, and the number of doses of laxatives that were needed between the control and submyofascial injection groups. $P$ values $<0.05$ were considered statistically significant. All statistical analysis was done using commercial software (Statcel4, OMS Publishing Inc., Japan).

\section{Results}

Of the 67 patients in total, 35 were in the control group and 32 were in the submyofascial injection group (Fig. 1). There were no significant differences in age, gender, weight, height, body mass index, or number of fusion levels in surgery between the two groups (Table 1). 


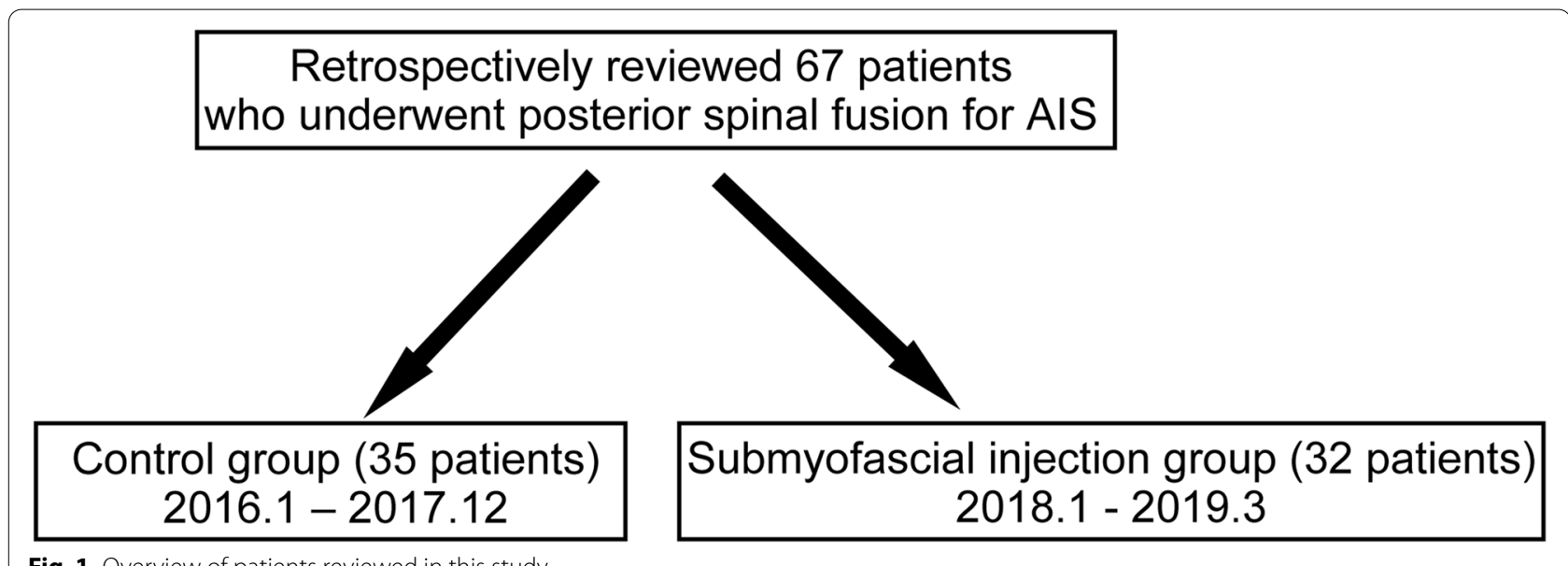

Fig. 1 Overview of patients reviewed in this study

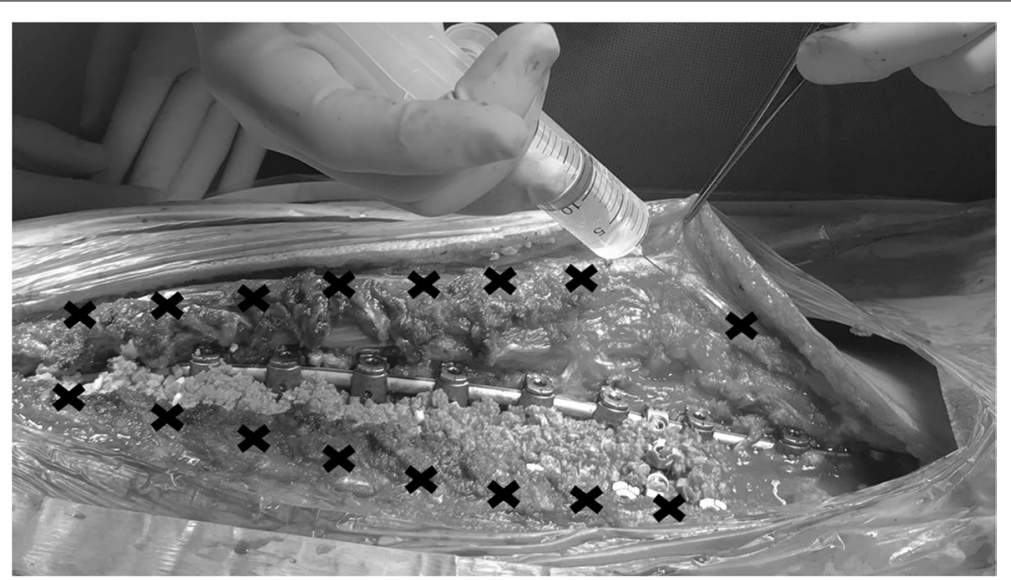

Fig. 2 The method of local injection with analgesic mixture. Bilateral infiltration of 1-2 $\mathrm{mL}$ of the mixture into the submyofascia at each disc levels

Table 1 The demographics of patients in this study

\begin{tabular}{llll}
\hline & Control & $\begin{array}{l}\text { Submyofascial } \\
\text { injection }\end{array}$ & $\mathbf{P}$ \\
\hline Number & 35 & 32 & \\
Age (y) & $15.0 \pm 2.3$ & $15.5 \pm 2.6$ & 0.44 \\
Gender (Women \%) & $32(91.4 \%)$ & $27(84.4 \%)$ & 0.37 \\
Height (m) & $1.57 \pm 0.08$ & $1.57 \pm 0.07$ & 0.99 \\
Weight (kg) & $48.3 \pm 9.4$ & $48.7 \pm 6.3$ & 0.85 \\
Number of fusion levels & $10.4 \pm 1.9$ & $9.3 \pm 2.3$ & 0.06 \\
\hline
\end{tabular}

\section{Evaluation of changes in postoperative pain and opioid} consumption

Although postoperative pain evaluated by NRS decreased over time in both groups, it was severe in the immediate postoperative period. The NRS values at $0 \mathrm{~h}, 12 \mathrm{~h}$ and $1 \mathrm{~d}$ after surgery in the submyofascial injection group were significantly lower than those in the control group. There was no longer a significant difference at $\geq 2 \mathrm{~d}$ (Fig. 3 ). The amount of fentanyl used was significantly less in the submyofascial injection group than in the control group at $24 \mathrm{~h}, 48 \mathrm{~h}$, and in total (Fig. 4).

\section{Comparison of postoperative activity}

The WRT was $3.3 \mathrm{~d}$ in the submyofascial injection group, significantly less than the $4.1 \mathrm{~d}$ in the control group (Fig. 5, $P=0.0007$ ).

\section{Adverse events and complications after surgery}

Of all 67 patients, 33 (49.2\%) patients had nausea requiring an antiemetic after surgery. In the control group, 19 (54.2\%) patients needed antiemesis, whereas, 14 (43.8\%) patients did in submyofascial injection group. This was 

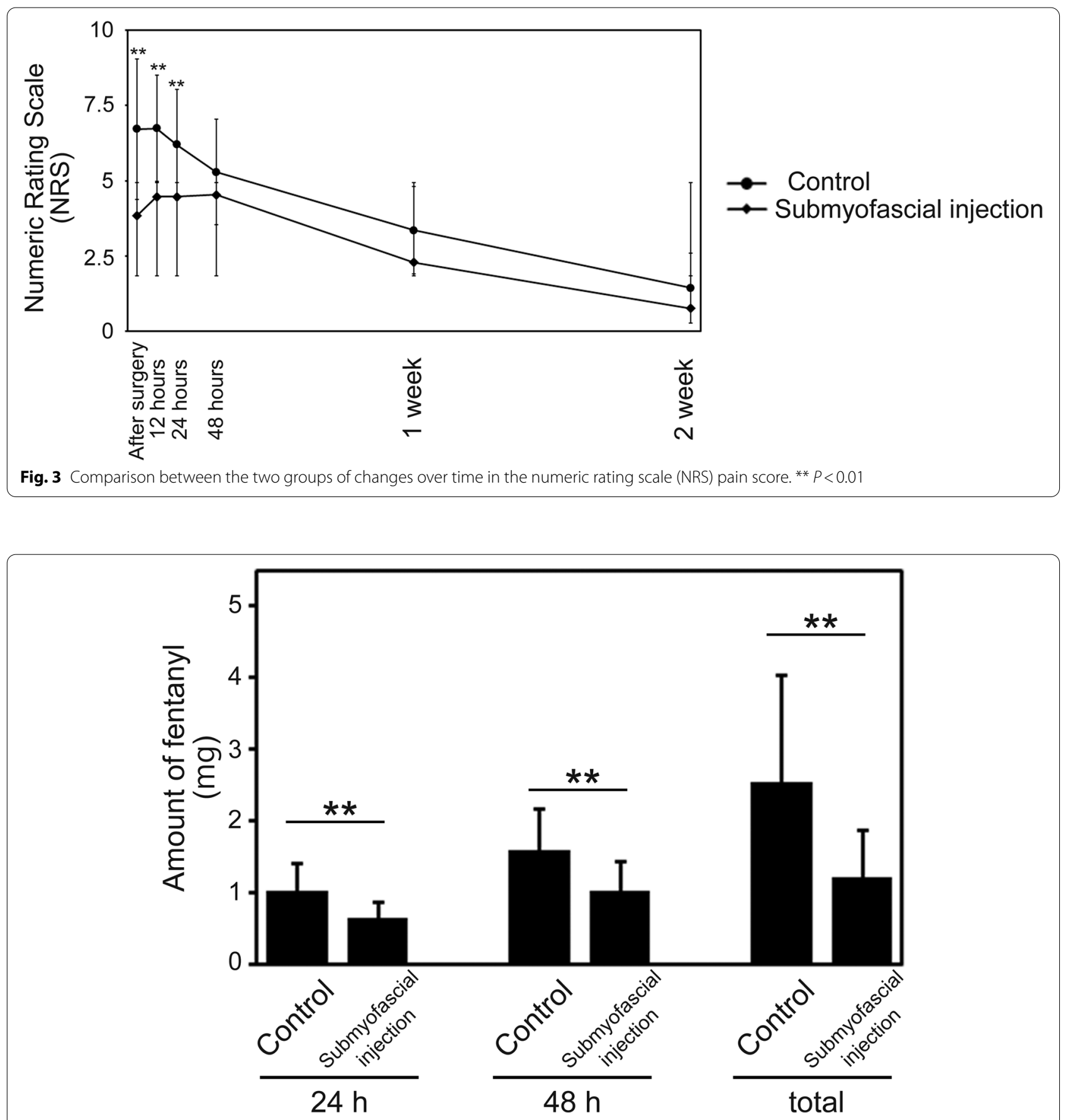

Fig. 4 Comparison between the two groups of the amount of fentanyl used at $24,48 \mathrm{~h}$ and in total after surgery. ${ }^{* *} P<0.01$

not significantly different between groups $(P=0.26$, Table 2).

There was no significant difference between groups concerning the recovery of transit materialized by the first defecation (mean $4.9 \mathrm{~d}$ after surgery). However, the mean number of times using a laxative was 1.3 times in the control group and 0.3 times in the submyofascial injection group, which was significantly less than in the control group $(P<0.01$, Table 2$)$.

There were no surgical site infections or neurological deterioration in any patient. There was no local anaesthetic systemic toxicity in the submyofascial injection group. In addition, there were no pseudarthroses following fusion surgery in any cases. 


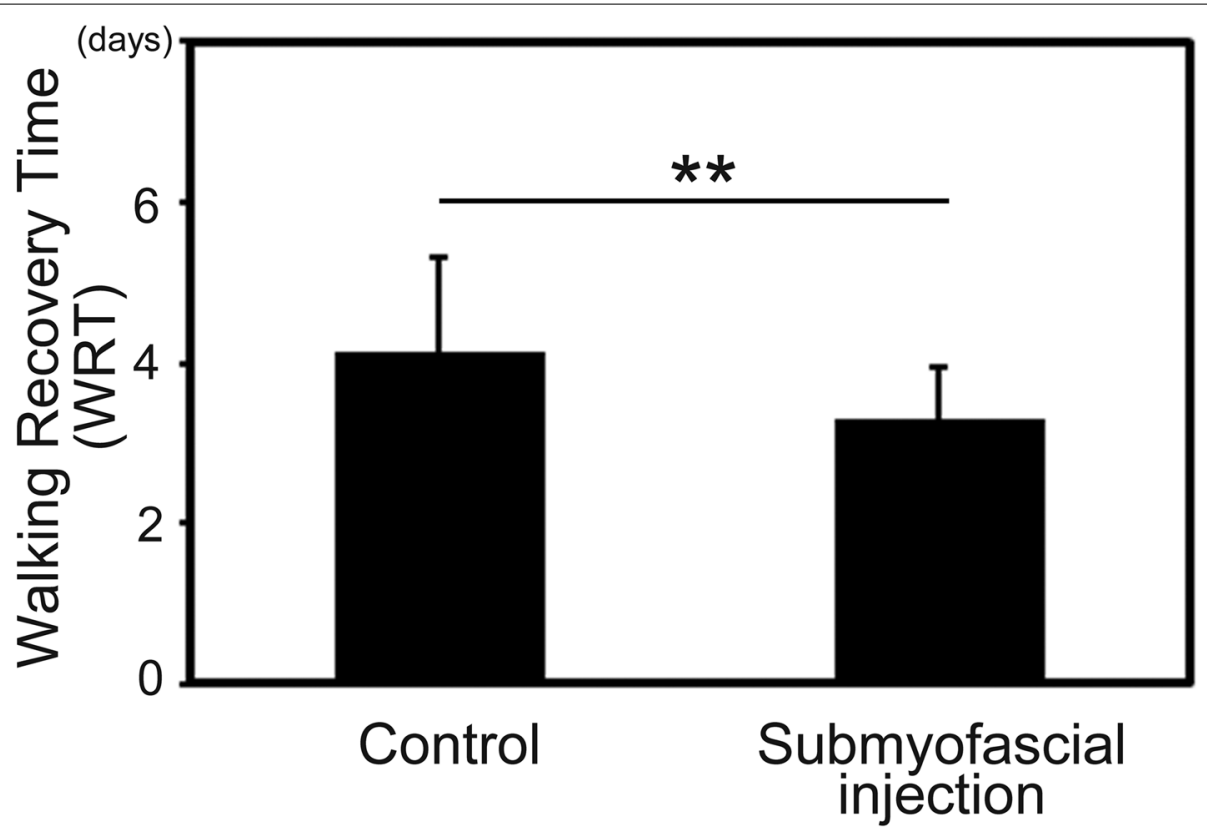

Fig. 5 The walking recovery time (WRT) defined as the number of days until the first ambulation after surgery in the two groups. ${ }^{* *} P<0.01$

Table 2 Comparison of treatment for adverse events

\begin{tabular}{llll}
\hline & Control & $\begin{array}{l}\text { Submyofascial } \\
\text { injection }\end{array}$ & $\mathbf{P}$ \\
\hline Use of antiemetic (times) & $1.0 \pm 1.2$ & $0.69 \pm 1.1$ & 0.26 \\
Duration of constipation (days) & $5.0 \pm 1.8$ & $4.7 \pm 1.3$ & 0.43 \\
Use of laxative (times) & $1.3 \pm 1.7$ & $0.3 \pm 0.6$ & $<0.01$ \\
\hline
\end{tabular}

\section{Discussion}

Postsurgical pain after posterior fusion for idiopathic scoliosis is severe, and use of opioids for severe pain may lead to adverse events such as nausea and constipation.

We performed submyofascial injections with a ropivacaine-epinephrine-dexamethasone mixture to reduce postoperative pain and opioid use and found that it significantly reduced postoperative pain on an NRS up to $1 \mathrm{~d}$ after surgery. It also significantly reduced opioid use and opioid-related adverse events and shortened the WRT.

The combined use of acetaminophen/NSAIDs for opioid-based postoperative analgesia is widely applied after orthopaedic surgery including spinal fusion. There have been some reports investigating the effect of acetaminophen/NSAIDs on postoperative analgesia, focusing on spinal fusion surgery for scoliosis [12-14]. They showed that acetaminophen/NSAIDs reduced postsurgical pain. However, Hiller et al. [12] reported that acetaminophen reduced postsurgical pain on a visual analogue scale but did not reduced opioid consumption. Analgesia with
acetaminophen/NSAIDs alone is usually inadequate, and other analgesic methods have been tried. The most common method of analgesia is IV-PCA with opioids [15], and we have used this method as the basis for postoperative analgesia. While the efficacy of IV-PCA has been widely reported [16], opioid-related adverse events such as PONV are of concern [17].

To achieve reductions in both postsurgical pain and opioid consumption, some methods combined with IV-PCA have been tried. First, epidural analgesia with/ without IV-PCA is also widely used for postsurgical pain in AIS undergoing posterior spinal fusion. One meta-analysis [18] showed that epidural analgesia had a better postoperative effect than IV-PCA. However, whether epidural analgesia reduced opioid-related adverse events such as PONV is still unknown [3, 19, 20]. Moreover, there have been concerns about adverse events such as respiratory depression; neurological changes, which are of the greatest concern to surgeons; oversedation; and catheter problems [21]. Intrathecal administration of opioids has been reported [22, 23]. Although this method contributes to analgesia and reduction of opioid use in the early postoperative period, respiratory depression can still be a problem [22]. Continuous wound infiltration has been recently used for postoperative analgesia [6-8]. This method is useful in reducing postoperative pain and opioid use, and although no serious complications have been reported, there have been concerns about the potential risk of surgical site infection associated with catheter 
placement [3]. We hypothesized that a single intraoperative infiltration of analgesics could eliminate this potential risk of infection and provide pain relief. In fact, local infiltration with analgesics for postsurgical pain is widely applied, especially in total knee arthroplasty and total hip arthroplasty, and its efficacy in reducing postsurgical pain and opioid consumption has been confirmed $[24,25]$. One-time submyofascial injection of a ropivacaine-epinephrine-dexamethasone mixture was useful for postsurgical pain in this study. The reasons for choosing the submyofascia as the injection site are that pain receptors are present in this layer [26] and that the posteromedial branch of the spinal nerve passes through the fascia. Recently, the analgesic effect of local injection in the submyofascia of the erector spinae has been reported [27].

This study has some limitations. First, this study was retrospective, and not a randomized controlled trial. A single surgeon at a single facility performed all the operations, so the variations in pain due to different surgical techniques were avoided. Second, the best mixture, concentration of local analgesics, and administration site (subcutaneous or submyofascial) are unknown. The effect of dexamethasone on postsurgical pain after posterior spinal fusion has been reported recently [28].

\section{Conclusion}

One-time postoperative local submyofascial injections with a ropivacaine-epinephrine-dexamethasone mixture reduced pain and the need for opioids after posterior spinal fusion. Injection of this mixture may be a useful adjunct to conventional analgesia in posterior spinal fusion for AIS.

\section{Abbreviations}

AIS: Adolescent idiopathic scoliosis; PCA: Patient-controlled analgesia; WRT :Walking recovery time; IV-PCA: Intravenous patient-controlled analgesia; NSAIDs: Non-steroidal antiinflammatory drugs; PONV: Postoperative nausea and vomiting; NRS: Numeric rating scale.

\section{Acknowledgments}

We thank Libby Cone, MD, MA, from DMC Corp. (www.dmed.co.jp < http:// www.dmed.co.jp/>) for editing a draft of this manuscript.

\section{Authors' contributions}

HM and SS wrote the main manuscript text and KK and YY collected the data. HM, SS and YK finalized the manuscript. All authors reviewed the manuscript. The author(s) read and approved the final manuscript.

\section{Funding}

This work was supported by JSPS KAKENHI Grant Numbers JP18K16613 and JP21K16679.

\section{Availability of data and materials}

The datasets used and/or analysed during the current study are not available publically due to privacy, but are available from the corresponding author on reasonable request.

\section{Declarations}

\section{Ethics approval and consent to participate}

This study was approved by the institutional review board of our university (the Ethics Committee of the University of Toyama). Informed consent was obtained from each patient and/or their legal guardian (For patients who less than 16 years old). This study were carried out in accordance with the Declaration of Helsinki.

\section{Consent for publication}

Not applicable.

\section{Competing interests}

There are no conflicts of interest to declare.

Received: 28 December 2021 Accepted: 28 February 2022

Published online: 04 March 2022

\section{References}

1. Kane WJ. Scoliosis prevalence: a call for a statement of terms. Clin Orthop Rela Res. 1997;126:43-6.

2. Lonstein JE. Scoliosis: surgical versus nonsurgical treatment. Clin Orthop Relat Res. 2006;443:248-59.

3. Seki $\mathrm{H}$, Ideno $\mathrm{S}$, Ishihara $\mathrm{T}$, et al. Postoperative pain management in patients undergoing posterior spinal fusion for adolescent idiopathic scoliosis: a narrative review. Scoliosis Spinal Disord. 2018:13:17.

4. Bajwa SJ, Haldar R. Pain management following spinal surgeries: an appraisal of the available options. J Craniovertebr Junction Spine. 2015;6:105-10.

5. Kessler ER, Shah M, Gruschkus SK, et al. Cost and quality implications of opioid-based postsurgical pain control using administrative claims data from a large health system: opioid-related adverse events and their impact on clinical and economic outcomes. Pharmacotherapy. 2013;33:383-91.

6. Ross PA, Smith BM, Tolo VT, et al. Continuous infusion of bupivacaine reduces postoperative morphine use in adolescent idiopathic scoliosis after posterior spine fusion. Spine. 2011;36:1478-83.

7. Reynolds RA, Legakis JE, Tweedie J, et al. Postoperative pain management after spinal fusion surgery: an analysis of the efficacy of continuous infusion of local anesthetics. Global Spine J. 2013;3:7-14.

8. Shrader MW, Nabar SJ, Jones JS, et al. Adjunctive pain control methods lower narcotic use and pain scores for patients with adolescent idiopathic scoliosis undergoing posterior spinal fusion. Spine Deform. 2015;3:82-7.

9. Yahara Y, Seki S, Makino H, et al. Three-dimensional computed tomography analysis of Spinal Canal length increase after surgery for adolescent idiopathic scoliosis: a multicenter study. J Bone Joint Surg Am. 2019;101:48-55.

10. Geck MJ, Macagno A, Ponte A, et al. The Ponte procedure: posterior only treatment of Scheuermann's kyphosis using segmental posterior shortening and pedicle screw instrumentation. J Spinal Disord Tech. 2007:20:586-93.

11. Seki S, Kawaguchi Y, Nakano M, et al. Rod rotation and differential rod contouring followed by direct vertebral rotation for treatment of adolescent idiopathic scoliosis: effect on thoracic and thoracolumbar or lumbar curves assessed with intraoperative computed tomography. Spine J. 2016;16:365-71.

12. Hiller A, Helenius I, Nurmi E, et al. Acetaminophen improves analgesia but does not reduce opioid requirement after major spine surgery in children and adolescents. Spine. 2012;37:E1225-31.

13. Munro HM, Walton SR, Malviya S, et al. Low-dose ketorolac improves analgesia and reduces morphine requirements following posterior spinal fusion in adolescents. Can J Anaesth. 2002;49:461-6.

14. Rosenberg RE, Trzcinski S, Cohen M, et al. The association between adjuvant pain medication use and outcomes following pediatric spinal fusion. Spine. 2017:42:E602-8.

15. Palmer GM, Pirakalathanan P, Skinner AV. A multi-Centre multi-national survey of anaesthetists regarding the range of anaesthetic and surgical 
practices for paediatric scoliosis surgery. Anaesth Intensive Care. 2010;38:1077-84.

16. Milbrandt TA, Singhal M, Minter C, et al. A comparison of three methods of pain control for posterior spinal fusions in adolescent idiopathic scoliosis. Spine. 2009;34:1499-503.

17. Poe-Kochert C, Tripi PA, Potzman J, et al. Continuous intravenous morphine infusion for postoperative analgesia following posterior spinal fusion for idiopathic scoliosis. Spine. 2010;35:754-7.

18. American Society of Anesthesiologists Task Force on Acute Pain Management. Practice guidelines for acute pain management in the perioperative setting: an updated report by the American Society of Anesthesiologists Task Force on acute pain management. Anesthesiology. 2012;116:248-73.

19. Klatt JW, Mickelson J, Hung M, et al. A randomized prospective evaluation of 3 techniques of postoperative pain management after posterior spinal instrumentation and fusion. Spine. 2013;38:1626-31.

20. Blumenthal S, Min K, Nadig M, et al. Double epidural catheter with ropivacaine versus intravenous morphine: a comparison for postoperative analgesia after scoliosis correction surgery. Anesthesiology. 2005;102:175-80

21. Sucato DJ, Duey-Holtz A, Elerson E, et al. Postoperative analgesia following surgical correction for adolescent idiopathic scoliosis: a comparison of continuous epidural analgesia and patient-controlled analgesia. Spine. 2005:30:211-7.

22. Tripi PA, Poe-Kochert C, Potzman J, et al. Intrathecal morphine for postoperative analgesia in patients with idiopathic scoliosis undergoing posterior spinal fusion. Spine. 2008;33:2248-51.

23. Gall O, Aubineau JV, Bernière J, et al. Analgesic effect of low-dose intrathecal morphine after spinal fusion in children. Anesthesiology. 2001;194:447-52.

24. Kurosaka K, Tsukada S, Seino D, et al. Local infiltration analgesia versus continuous femoral nerve block in pain relief after total knee arthroplasty: a randomized controlled trial. J Arthroplast. 2016:31:913-7.

25. Kuchálik J, Granath B, Ljunggren A, et al. Postoperative pain relief after total hip arthroplasty: a randomized, double-blind comparison between intrathecal morphine and local infiltration analgesia. Br J Anaesth. 2013;111:793-9.

26. Hoheisel U, Taguchi T, Treede RD, et al. Nociceptive input from the rat thoracolumbar fascia to lumbar dorsal horn neurones. Eur J Pain. 2011:15:810-5.

27. Forero M, Adhikary SD, Lopez H, et al. The erector spinae plane block: a novel analgesic technique in thoracic neuropathic pain. Reg Anesth Pain Med. 2016;41:621-7.

28. Fletcher ND, Ruska T, Austin TM, et al. Postoperative dexamethasone following posterior spinal fusion for adolescent idiopathic scoliosis. J Bone Joint Surg Am. 1807-1813;202:102.

\section{Publisher's Note}

Springer Nature remains neutral with regard to jurisdictional claims in published maps and institutional affiliations.

Ready to submit your research? Choose BMC and benefit from:

- fast, convenient online submission

- thorough peer review by experienced researchers in your field

- rapid publication on acceptance

- support for research data, including large and complex data types

- gold Open Access which fosters wider collaboration and increased citations

- maximum visibility for your research: over $100 \mathrm{M}$ website views per year

At BMC, research is always in progress.

Learn more biomedcentral.com/submissions 